\title{
Zusätzliche Gabe von Delamanid bei resistenten Stämmen kosteneffektiv?
}

\author{
Auch wenn die weltweiten Inzidenzraten der Tuberkulose (TB) \\ rückläufig sind, stellen multiresistente Stämme weiterhin eine \\ große Herausforderung dar. R. Diel et al. gingen nun der Frage \\ nach, ob in diesen Fällen die zusätzliche Gabe von Delamanid \\ zu einem Regime aus 5 Tuberkulostatika kosteneffektiv ist. \\ Resp Med 2015; 109: 632-641
}

Die Autoren berechneten hierzu die inkrementelle Kosteneffektivität der Behandlung mit Hilfe eines Markov-Modells. Zugrunde legten sie die Daten der 24-Monats-Ergebnisse von den 3 Studien, die bisher zu Delamanid durchgeführt worden waren. Als Modell diente eine hypothetische Kohorte von 1000 Patienten im Durchschnittsalter von 38 Jahren, die an einer TB durch multiresistente Stämme litten. Die Behandlung bestand aus einem Regime von 5 Medikamenten in Kombination mit Delamanid oder lediglich der Fün- ferkombination. Die Autoren verglichen beide Varianten über einen Zeitraum von 10 Jahren und bestimmten die Kosten pro qualitätsadaptiertem Lebensjahr (QALY) sowie die bezüglich gesundheitlicher Einschränkungen angepassten Lebensjahre (DALY) aus gesellschaftlicher Perspektive.

Basierend auf den aktuellen Preisen beliefen sich die diskontierten Gesamtkosten pro Patient für die Variante Fünferkombination plus Delamanid auf $142732 €$ und für die Fünferkombination alleine auf
$150909 €$. Die gesamten diskontierten QALYs pro Patient lagen bei 8,47 für die Kombination mit Delamanid und bei 6,13 für die Fünferkombination alleine. Entsprechend erwies sich die Zugabe von Delamanid gegenüber der alleinigen Therapie mit der Kombination aus 5 Tuberkulostatika als überlegen und konnte sowohl $8177 €$ an Kosten einsparen als auch 2,34 zusätzliche QALYs erzielen. Delamanid fand sich in $73 \%$ der probabilistischen Sensitivitätsanalysen als kostensparend im Vergleich zur Fünferkombination alleine und als $100 \%$ kosteneffektiv bei einer „Willing-topay“-Schwelle von $10000 €$.

Fazit

Unter den Bedingungen in Deutschland ist die Zugabe von Delamanid zu einem Regime aus 5 Tuberkulostatika bei multiresistenten uberkulosestämmen wahrscheinlich kostensparend im Vergleich zu einer Fünferkombination alleine. Diese Ergebnisse sprechen nach Angaben der Autoren deutlich für die Gabe von Delamanid bei diesen Patienten.

Dr. Johannes Weiß, Bad Kissingen

\section{Mukoviszidose in bundesweites Neugeborenen-Screening aufgenommen}

Jedes Neugeborene in Deutschland kann ab Frühjahr 2016 auf Mukoviszidose untersucht werden. Das hat das oberste Beschlussgremium der Gemeinsamen Selbstverwaltung von Ärzten, Psychotherapeuten, Krankenhäusern und -kassen sowie der Gemeinsame Bundesausschuss entschieden. Eine Pilotstudie am Universitätsklinikum Heidelberg, die von der DietmarHopp-Stiftung und vom Deutschen Zentrum für Lungenforschung finanziert wurde, hatte gezeigt: Der Bluttest entdeckt die Krankheit zuverlässig und kann im Rahmen des Neugeborenen-Screenings angewandt werden. Die kleinen Patienten können so von Anfang an medizinisch betreut und frühzeitig behandelt werden. Dies soll helfen, die körperliche Entwicklung und Lungenfunktion der betroffenen Kinder zu verbessern. „Das ist ein riesiger Erfolg und hilft vor allem den betroffenen Kindern und Familien“, berichtet Prof. Georg Hoffmann, Heidelberg. „Ich freue mich über je- den Jungen und jedes Mädchen, denen das Neugeborenen-Screening hilft, eine treffende Diagnose zu erhalten. Bei den meisten Krankheiten gilt: Je früher sie erkannt und therapiert werden, desto besser. Die Ausweitung des Screenings zeigt, dass sich die Investition in das Heidelberger Früherkennungsprogramm lohnt“, sagt Stifter Dietmar Hopp.

Jährlich kommen in Deutschland rund 200 Kinder mit Mukoviszidose zur Welt; ungefähr eines von 3300 Neugeborenen ist betroffen. Fehler an einer bestimmten Stelle im Erbgut, im genetischen Bauplan des Proteins CFTR (Cystic Fibrosis Transmembrane Conductance Regulator), lassen die Sekrete in Lunge und Verdauungstrakt austrocknen und führen zu schweren fortschreitenden Funktionsstörungen von Lunge, Bauchspeicheldrüse, Leber und Darm. Zäher Schleim verstopft die Atemwege. Dies begünstigt chronische Infektio- nen sowie Entzündungen und führt zu bleibenden Lungenschäden. Eindeutige klinische Symptome zeigen sich oft erst im Alter von mehreren Jahren. Bis dahin unentdeckte Veränderungen, vor allem in der Lunge, sind dann zum Teil nicht mehr rückgängig zu machen. Die Untersuchung auf Mukoviszidose erfolgt zum selben Zeitpunkt und aus der gleichen Blutprobe wenige Tropfen aus der Ferse des Kindes genügen, wie das bereits etablierte Neugeborenen-Screening zeigt. Basis des Screenings auf Mukoviszidose ist ein biochemischer Test, den die Wissenschaftler für das Screening adaptiert haben. Mit Einverständnis der Eltern wird das Blut des Kindes auf insgesamt 15 Krankheiten untersucht: 13 Stoffwechselerkrankungen und 2 Hormonstörungen. Eines von 1200 Kindern leidet an einer der Krankheiten, nach denen im Screening gefahndet wird. Der sofortige Beginn der Behandlung sowie die engmaschige Betreuung verhindern bei den meisten Betroffenen lebensbedrohliche Stoffwechselkrisen und damit Schäden am Gehirn und an anderen Organen.

\section{Nach einer Mitteilung des} Universitätsklinikums Heidelberg 